\title{
Are Stellar Kinds Natural Kinds? \\ A Challenging Newcomer in the Monism/Pluralism and Realism/Antirealism Debates
}

\begin{abstract}
Stars are conspicuously absent from reflections on natural kinds and classifications, with gold, tiger, jade, and water getting all the philosophical attention. This is too bad for, as this paper will demonstrate, interesting philosophical lessons can be drawn from stellar taxonomy as regards two central, on-going debates about natural kinds, to wit, the monism/pluralism debate and the realism/antirealism debate. I show in particular that stellar kinds will not please the essentialist monist, nor for that matter will it please the pluralist embracing promiscuous realism à la Dupré. I conclude on a more general note by questioning the relationship between taxonomic scientific practice and philosophical doctrines of natural kinds.
\end{abstract}

1. Introduction. "Species are evidently not arbitrary like the grouping of the stars in constellations": Darwin ([1859] 1962, 411) did not have apparently a high opinion of stellar taxonomy, nor did the French mathematician and philosopher A. A. Cournot, for whom constellations were a typical example of artificial groups of things ([1851] 1975, 199-208). But stellar taxonomy has come a long way since Darwin and Cournot's time. Yet, it is still remarkably absent from reflections on natural kinds and classifications, with gold, tiger, jade and water getting all the philosophical attention. It's a pity for, as we shall see, the practice and achievements of stellar taxonomy raise fruitful challenges to the current main philosophical standpoints on natural classifications. Moreover, stellar classifications are representative of what many taxonomic enterprises are about in science today, that is, coming up with kindmembership conditions that define epistemically fruitful groupings of entities whose diversity is revealed (or even created) by scientific investigation, rather than trying to discover the hidden essence of antecedently recognized kinds. In short, from a taxonomic point of view, stars share with microbacteria, subatomic particles, nanotubes and other members of the bestiary of microbiology and microphysics, a lack of "quotidian prehistory" (Daston 2000): they were not part of our reality - and therefore not the subject of ordinary-language classifications - before they became subject of scientific inquiry and classifications ${ }^{1}$. Not surprisingly then, I won't have

$1 \quad$ Isn't that plainly wrong for stars? Surely stars were part of our reality before they became subject of scientific inquiry and classifications. But as explained in the next section, their ontological status radically changed with the advent of spectroscopic observations that revealed their diversity, so that the issue of the relationship between antecendently recognized kinds and scientific kinds does not even arise for stars, contrary to the cases of biological species or chemical kinds. 
much to say about traditional issues of reference of ordinary language natural-kind names. On the other hand, my discussion of stellar kinds will be directly relevant to two other central, on-going debates about natural kinds and classifications, to wit, the monism/pluralism debate and the realism/antirealism debate.

On the face of it, stellar taxonomy does not seem to be a very monist-friendly domain. Take one of the brightest stars of the Northern hemisphere, Vega in the Lyra constellation, and ask astrophysicists what kind of star Vega is. Commonly known as a "A0 V" star, that is, a relatively hot, slightly bluish "main sequence dwarf", Vega is also classified by astrophysicists observing in the far infrared part of the electromagnetic spectrum as a " $1 \mathrm{n}-18$ " star, that is, a star with no remarkable spectral feature in this domain of wavelength, and for those studying how the light emitted by a star varies, Vega is known as a "Delta scuti" type of star, that is, a kind of pulsating variable star.

My aim in this paper is to investigate how this taxonomic pluralism should be interpreted and which metaphysical and epistemological lessons can be drawn from it about natural kinds and classifications. I start with a bit of history about stellar classifications. Then a (shallow) plunge in the practice of grouping stars will allow us to grasp the sources of stellar taxonomic pluralism. Along the way, comparisons with two familiar cases - the classification of chemical elements and the classification of living organisms-, will bring out the specificities of the stellar case. I then discuss whether the stellar world comes prepacked with any objective divisions, putting on the carpet both essentialist standpoints on natural kinds and non-essentialist, realist ones, such as Dupré's promiscuous realism (Dupré, 1993). On a more general note, I conclude by questioning the relationship between taxonomic scientific practice and philosophical doctrines of natural kinds.

2. Historical markers. As any lover of the celestial wonders knows, nothing looks more like a star than another star... even when observed with a telescope ${ }^{2}$. Not surprisingly then, the ordering of stars had long been restricted to the rudimentary task of noting their positions and estimating their apparent brightness. The first systematic

2 That might be the reason why, by the way, stellar classifications have not yet received much philosophical attention, in spite of their rich history and crucial role in astrophysics: stellar diversity does not stare in the face as does the diversity of, say, plants, animals, minerals or chemical substances. No wonder then than most philosophical discussions about carving up the world have been so far restricted to the terrestrial world. 
survey is attributed to Hipparchus who classified 1080 stars (one fifth of the number of stars visible to the naked eye) in 49 constellations ordered by their positions on the $\mathrm{sky}^{3}$. Three centuries later, Ptolemy listed 1022 stars divided in six categories of apparent brightness. In the XVIIIe and XIXe century, the number of stars ordered by positions and magnitude increased significantly. Let us mention the remarkable work of Nicolas Louis de La Caille who, in 1750, went with his instruments to the Cape of Good Hope to catalog ten thousand stars of the Southern Hemisphere, and Herschel's famous Urano Metria Nova published in 1843, and cataloging 3256 stars of the Northern Hemisphere. But the really significant turn in stellar classification came with the development of stellar spectroscopy, directly linked to the development of spectroscopy in the laboratory.

On the theoretical side, G. Kirchoff and R. Bunsen (building on previous work by J. Foucault) had laid down by the 1860's the two basic principles of spectrum analysis. ${ }^{4}$ Astronomers first applied these principles to the analysis of the solar spectrum and, thanks to progress made on the observational side in photography, they were soon able to apply them as well to the analysis of the much fainter stellar spectra. By 1880, the spectra of several thousands of stars had been collected, revealing their diversity: stellar spectra turned out to differ both in the position of their bright lines and in their "color" (which reflect the overall distribution of energy), thereby suggesting important differences in the physical properties of the stars. Then arose the issue of the origin of this spectral diversity. Was it due to differences in temperature, in chemical composition, or both? Did different stellar spectra correspond to different stages in the evolution of a star? The intricate arguments exchanged by the main protagonists of the debate at that time - the Jesuit A. Secchi, H. C. Vogel, J. N. Lockyer and W. Huggins involved a rather fascinating mixture of embryonic theoretical knowledge of the behavior of gaseous spheres, empirical considerations mainly based on comparisons with spectra obtained in the laboratory,

3 The story goes that Hipparchus was at Rhodes observing the sky when he witnessed the apparition of a very bright star. He then decided to come up with a catalogue of stars that could serve as a reference to record this kind of event. For more on these early catalogues, see Pannekoek 1961.

$4 \quad$ In short, the first principle stated that solids and liquids typically produce continuous spectra in the visible, whereas gases produce characteristic emission lines. The second principle stated that when a source producing a continuous spectrum is seen through a cold gas, the wavelengths of its absorption lines correspond to the wavelengths of the emission lines produced by the gas when heated. 
personal convictions such as the reluctance to give up the idea of cosmic uniformity, and influences of the epistemic context of the time, in particular the newly born theory of evolution, from which general ideas of evolution were directly (albeit loosely) imported. ${ }^{5}$

The path leading from this early debate to what is considered today as the correct interpretation of differences in stellar spectra covered several decades marked both by progress in instrumentations and by theoretical breakthroughs in the general understanding of spectra (especially Megh Nad Saha's work, in the 1920's, on the ionisation of chemical elements). This path having been quite torturous, I can only refer to DeVorkin (1978) and Ruphy (1997) for the historical details of its many turns. What I want to emphasize here is that spectroscopy did not only reveal the diversity of the stars but also triggered a radical mutation in their ontological status. Stars definitely lost their status of incorruptible objects inherited from the Ancients to progressively become objects having a "natural life", that is, objects evolving through different phases, displaying diverse physical properties. For a long time restricted to the study of positions and movements of stars, the task of the astronomer progressively came to focus on the understanding of their physical properties and evolution. And, most importantly for our purpose, this mutation went hand in hand with a radical mutation of the classificatory act.

So far stars had been classified by apparent properties: the positions listed in catalogs such as Ptolemy's and Herschel's were apparent positions, corresponding to the projection on the celestial vault of the "real" positions of the stars in the Galaxy, and brightness, corresponding to the quantity of light received by us, not to the quantity of light emitted by the star, called absolute or intrinsic brightness. When spectral diversity suggested differences in physical properties, stellar taxonomists became more ambitious: their aim was henceforth to go beyond the appearances by building classificatory schemes based on intrinsic characters.

The classification proposed by Secchi in the 1870's - the first one to be based on spectral features - inaugurated this new ambition. Secchi's observations, as well as observations by Vogel, Lockyer and Huggins had led to the conclusion that spectral diversity could be organized by grouping stars together in a very limited number of

$5 \quad$ Further analysis, in Bachelardian terms, of the influence of contextual values on the genesis of stellar classifications can be found in Ruphy 2006. On early interpretations of spectral classifications, see also Shapere 1987 and Pannekoek 1961. 
categories, according to their color. Secchi assorted the several thousands of stars for which spectra were available at that time in three categories: "class I" contained the blue stars, "class II" the yellow stars such as the Sun and "class III" the red stars. The collection of more stellar spectra at higher dispersion soon revealed the inhomogeneity of Secchi's categories, thereby calling for their revision. But the essential spirit of modern stellar classifications was already there: assort stars not according to how they appear to us, but according to their intrinsic properties. Those familiar with the history of scientific classifications may recognize a similar mutation, roughly at the same time, that is, in the second half of the XIXe century, in disciplines such as crystallography and mineralogy where taxonomy was also the backbone of everything else.

In the wake of Secchi's work, early spectral classifications were onedimensional: stars were assorted into kinds according to their "spectral type". In 1907, the Danish astronomer Ejnar Hertzsprung and the American astronomer Henry Norris Russell established by independent means an empirical relation between the spectral type and the class of luminosity ${ }^{6}$. This empirical relation translated into the localization of stars in certain specific areas of a two-dimensional diagram (now called the Hertzsprung-Russell diagram or, in short, the HR diagram) where stars were distributed by these two parameters. Most stars (including our Sun) found a place along a diagonal now called the "main sequence" and received the label "dwarf”. Brighter stars divided up into different types of categories now called "giants", "supergiants", etc (see Fig. 1). Stars thus turned out to significantly differ not only by spectral type but also by their class of luminosity, which reflects differences of volumes and densities. Hence the adoption of this intrinsic property as a second taxonomic criterion.

The epistemic importance of the HR diagram cannot be overstated. Not only did it pave the way to the currently most widely used two-dimensional classifications ${ }^{7}$, but also this putting into a table of the stellar multiplicity has been

6 The spectral type of a star is defined by the shape of its spectrum in the visible part of the electromagnetic spectrum and by the position and brightness of some emission lines. It is mainly governed by the temperature of the star. The class of luminosity is defined by the ratio of intensity of certain lines located in the visible part of a stellar spectrum and varies with the density of the star.

7 The most comprehensive classification systems are, on the one hand, the "Morgan Keenan" or "MK" system of spectral classification and, on the other hand, photometric classifications based on color indices. These systems are two-dimensional systems based on 
fruitful in many other ways. It revealed for instance the existence of families of stars having very different volumes and corresponding to different stages of stellar evolution, and still plays a key role in the understanding of the physical nature and the evolutionary stage of newly observed stars. Since its first elaboration at the beginning of the last century, the HR diagram has been revised several times, but its main structure has remained essentially unchanged, so that the very history of the modifications of the diagram displays its stability and epistemic virtues. For all that, astrophysicists could hardly have given into the metaphysical hubris of believing that they had come up with the ultimate right way of carving up the celestial world. And indeed, the two-dimensional system grounding the HR diagram, based on spectral features observable in the visible part of the electromagnetic spectrum, was completed by other taxonomic systems based on different parameters. In the late 1970' and 1980', with the launch of satellites embarking detectors working in other domains of wavelength (in particular in the infrared and the ultraviolet), astronomers began to design independent classificatory schemes based on spectral features observable in these newly accessible domains. For certain types of stars, an alternative taxonomic strategy was to combine these spectral features with "visible" spectral features and develop classificatory systems whose dimensions were superior to two. ${ }^{8}$ Hence a multiplication of wavelength-dependent taxonomic systems. It is worth noticing, though, that due to practical observational limits (and budgetary constraints...), the development of independent classificatory schemes in various regions of the electromagnetic spectrum, or the refinement of certain existing categories by the use of additional criteria, concern only a very limited number of stars compared to the number of stars classified in a HR diagram. In other words, there are large differences as regards the comprehensiveness of the various taxonomic systems, with systems based on visible features being by far the most comprehensive. This predominance is easily explained. The Earth atmosphere happens to be transparent and we, human observers, happen to see in this domain of wavelength. Not surprisingly then, the most

parameters relative to the visible part of the electromagnetic spectrum. For more detail see Jaschek and Jaschek (1990).

$8 \quad$ Note that those systems remain local in the sense that each is a refinement of some specific chunk of the HR diagram. Adding for instance a UV based citerion can be done only for certain homogeneous classes in the visible, such as hot blue stars. The same criterion cannot be used for another chunk of the HR diagram, say, red giant stars, for those stars do not exhibit features in the UV that would allow to classify them according to this criterion. In that case, an additionnal infrared criterion is needed. 
comprehensive classification systems are relative to this domain. But one can easily imagine that alien astrophysicists endowed with a sharp view in, say, the infrared, would have come up with large-scale, comprehensive classifications based on infrared properties rather than visible ones. Hence the contingency of the current taxonomic landscape.

My skimming through the intricate history of stellar taxonomy has left aside the development of taxonomic systems of limited comprehensiveness, based on properties that are not read off stellar spectra, such as the classification of stars based on the variation of their luminosity. But it should suffice to make vivid the following historical tendency: the more mature stellar astrophysics has become (both by getting huge amount of complementary observations in various domains of wavelength and by improving theoretical understanding of stellar physics), the more taxonomic systems have multiplied.

To make sense of this tendency to pluralism and grasp its philosophical imports, we need now a (shallow) plunge in the details of the practice of grouping stars. We need in particular a closer look at the kind-membership conditions and the resulting philosophically challenging characters of stellar kinds.

\section{The art and quirks of grouping stars}

\subsection{Stellar structuralism. Stars are grouped together in a class when they exhibit} features "similar enough" to those of a standard star picked up to define the class. But of course, not any feature will do as a similarity parameter. For instance, in spite of the fact that this intrinsic property is known for a very large number of stars, stars are not classified by their proper motion, for the same reasons that animals are not sorted out by, say, their number of hairs, or chemical substances by their color: what stellar taxonomists share with their confrères in other scientific domains is a search for "privileged" or taxonomically "significant" similarity parameters. My putting these epithets between inverted comas follows a widespread habit in philosophical discussions about natural kinds and classifications, which conveys a crucial ambiguity of the use of the terms. In what sense can similarity parameters be said to be "privileged"? A metaphysical reading of the term usually refers to some form of essentialism. For less metaphysically inclined philosophers, the reading may be more epistemological: a set of similarity parameters is privileged to the extent that it defines 
epistemically fruitful groupings (i. e. lending themselves to generalizations and predictions, playing a role in causal explanations, etc.). Actually, it is hardly overstated to claim that the main lines of disagreement between various standpoints on natural kinds and classifications (i.e. monism, pluralism, realism, antirealism and some combinations of those four) boil down to divergent views of what "privileged" means. But until section 5 where I'll ask whether stellar kinds are natural kinds and discuss these divergent views more extensively, I'll remain neutral as regards the appropriate reading of the notion in the astrophysical context, and stick to the description of kind-membership conditions in the scientific practice of sorting out stars.

So what are the similarity parameters that are taken as "privileged" by stellar taxonomists? The brief aforementioned historical markers gave us a hint: early taxonomic systems were based on spectral features that were measurable for a large number of stars and that were deemed significant from a theoretical point of view. Such a combination of practical and theoretical considerations later continued to shape taxonomic enterprises and still constrains today new classificatory developments.

Astrophysicists want to know how stars form, evolve and die. Their theoretical understanding of the behavior of gaseous spheres tells them that parameters such as temperature, density or mass loss are determinant parameters in stellar evolutionary processes, whereas proper motion or distance from the Earth are not; hence their choice of the former, and not the latter, as taxonomic parameters. In short, kindmembership is conferred by structural properties central for explaining a large variety of stellar behaviors.

I've mentioned taxonomic parameters such as spectral type, mass loss, class of luminosity, temperature, etc. Following scientific practice, I have mixed together two types of parameters, but the distinction between the two types is worth being made explicit for epistemological purpose. Taxonomic intrinsic properties may be directly or indirectly observable. The spectral type of a star is a directly observable - or "manifest" property, for it can be "directly" read off its spectrum". By contrast, a

\footnotetext{
9 The inverted commas here draw attention to the fact that "direct" observation never means "brute" observation in astrophysics. The observer must always correct the signal received from the alterations it underwent when light passed through interstellar dust and the
} 
structural property such as temperature is a non-directly observable property: quite obviously, astrophysicists do not go around space with a thermometer to measure it; they have to use elaborate models of stellar atmosphere to derive temperature from manifest properties. And the same goes for structural properties such as the radius of a star, its rotation speed, its magnetic field or its mass loss. More generally, manifest properties are properties of the light emitted by a star, given that no measurement can be made in situ. Structural properties are then derived from manifest ones via more or less complex theoretical models. Systematic links have been for instance established via theoretical models between spectral type and temperature, and also between class of luminosity (directly read off a spectrum) and intrinsic brightness or density. ${ }^{10}$ Observational access to structural properties is thus mediated by theoretical modeling.

This form of theory-ladenness has two consequences for the taxonomist. First, it leads to a "two-layer" structure of taxonomic systems, with a first layer based exclusively on manifest properties and often described as the "empirical" classificatory system, and a second layer related to the first via calibrated theoretical models (in practice, the two layers are often mixed). If the empirical layer is rather stable, astrophysicists have to live with a certain degree of instability of the second layer, according to the way calibrations are revised in light of new theoretical and observational developments. Second, the theory-ladenness of the observational access to structural properties somehow limits the possibilities of choice of taxonomic parameters. Not all structural properties deemed significant from a theoretical perspective can be systematically related to some manifest properties and used as a taxonomic parameter. For instance, astrophysicists interested in stellar evolution would be very happy to be able to group stars according to their mass. Unfortunately, except for a small fraction of stars, they do not have reliable theoretical models that systematically relate this structural property to manifest (i.e. directly observable) properties. In short: both practical and theoretical considerations limit the possibilities of choice of taxonomic parameters. A structural property deemed significant must be linkable to some manifest properties via a theoretical model, and those manifest properties must be measurable for a large number of stars.

Earth atmosphere, just to mention the main tricky distorting effects that astrophysicists have to cope with.

10 So that a HR diagram can be graduated in any combination of these parameters, that is, spectral type or temperature $v s$. class of luminosity or density or intrinsic brightness. 


\subsection{Resolution Dependency. Most stellar structural parameters (and their} corresponding manifest properties) vary continuously from one star to another. Therefore, in the stellar bestiary, there does not exist any level of genuine discontinuity above the level of individual stars. In other words, by analogy with the notion of "infimic species" defined by Ellis $(2002,57)$ as "a species that has no subspecies, [and that] is ultimately specific" ", an astrophysicist cannot expect to come up with "infimic stellar kinds" (more on the important consequences of that point in the last section when I discuss essentialism and realism about kinds). Let's spell out why. Stellar kinds are defined by cutting up the observational continuity into boxes. We have seen that stars are grouped together in a class when they exhibit structural features "similar enough" to those of a standard star picked up to define the class and that those features vary continuously from one star to another. For a given set of similarity parameters, whether a star is similar enough to the standard one depends on the resolution of the observations used by the classifiers. In other words, the degree of similarity between two stars remains dependent on instrumental resolution. In the case of classifications based on spectral features, the grouping of stars is bound to the dispersion of the spectra. Increasing spectral dispersion reveals inhomogeneities in a class homogeneous at lower dispersion, thus calling for the creation of subclasses, subsubclasses, etc., to keep a satisfying homogeneity in the basic taxonomic unit. Classes of stellar objects being defined by the cutting up of the observational continuity, the more resolution you have, the more fine-grained can be the cutting. Hence what I call the "resolution dependency" of stellar classifications. ${ }^{12}$

3.3. Vagueness. Another straightforward consequence of stellar structure being defined by continuous parameters (such as temperature and density) is the vagueness of the similarity relations between two stars. As a result, stellar kinds do not have sharp boundaries and a star may be classified as intermediate between two kinds.

\footnotetext{
11 Good candidates for the status of infimic kinds are for instance atoms in their stable states.

12 The most comprehensive 2D spectral taxonomic system, the MK system, requires spectra at a dispersion of $115 \mathrm{~A}^{\circ} / \mathrm{mm}($ at $\mathrm{H} \gamma)$. Spectra at higher dispersion have been obtained for stars of a certain spectral type (O stars for instance) leading to the division of the corresponding "MK box" into finer boxes. For more detail on what astrophysicists often call "precision spectral classification", see Gray 1994.
} 
Consider the two-dimensional taxonomic system based on spectral types (labeled by a capital letter and a numeral) and classes of luminosity (labeled by a Roman numeral). A "K0 V / K1 V" star is a star whose spectral type and class of luminosity (and the corresponding structural properties) are as much "similar enough" to those of the standard star defining the kind " $\mathrm{K} 0 \mathrm{~V}$ " as they are to those of the standard star defining the kind "K1 V".

As regards the vagueness of the similarity relations, stellar kinds resemble to a certain extent molecular kinds. Because of isomerism, to define molecular kinds, similarity of chemical element composition must be supplemented by similarity of molecular structure. But as Hendry (2006) reminds us, sameness of molecular structure is a vague relation since molecular structure is defined in terms of variables internuclear distances and angles between bonds- that vary continuously. As a result, "interatomic geometry will [...] group molecules into vague-bounded, overlapping clusters of similar structures.” (Hendry 2006, 869)

\subsection{Taxonomic nomadism. Another important feature of stellar taxonomy is} the fact that a star's classification is not a permanent matter: properties on which stellar classifications are based are transitory properties. A star does not have the same spectral type, class of luminosity, mass loss, etc., throughout its life. Consider the Sun's classification in the familiar 2D spectral taxonomic system: its localization in a HR diagram will change when the Sun evolves. Currently classified as a G2 V star (i.e. a yellowhish dwarf star located on the main sequence), it will move onto the red giant branch, before ending up in the white dwarf area of the diagram. I will call this specificity "taxonomic nomadism". Note that the pace of stellar taxonomic nomadism is rather slow. The Sun will spend altogether more than 8 billion years in its current category before moving on to another one.

Is taxonomic nomadism an idiosyncrasy of the stellar world? On the face of it, stellar taxonomic nomadism has no strict equivalent in the biological realm. An organism's classification is a permanent matter: a new-born tiger is already a tiger and remains so during its entire developmental course (whatever the kind-membership conditions are - essentialist, phylogenetic, interbreeding, etc.). Only when species are considered as individuals rather than sets or classes (Ghiselin 1974; Hull 1978) may an analogy be considered. In that case, both species and stars are indeed the subject of 
evolutionary processes. Considered as spatiotemporally restricted continuous entities, a species may evolve into another species. But the analogy remains limited, for taxonomic nomadism does not operate at the same ontological level in both cases. Stellar taxonomic nomadism concerns the individual members of stellar classes, whereas biological taxonomic nomadism does not concern the parts (the individual organisms) that form the wholes (species), but the wholes themselves. Actually, the closest case to stellar taxonomic nomadism is the phenomenon of natural transmutation of chemical elements, where a member of a chemical kind, say, thorium, naturally becomes a member of another chemical kind (radium).

Taxonomic nomadism may turn out to be an embarrassment for certain philosophical standpoints on natural kinds (more on that in the last section), but it is a feature much appreciated by astrophysicists. Here's why. Given the timescale of stellar evolution, astrophysicist cannot study evolutionary processes by monitoring the evolution of individual stars. To learn about the different evolutionary phases - in particular how long a star will spend in each of these phases-, they compare how many stars belong to each of the associated stellar kinds. From the statistical repartition of stars into kinds, they are then able to derive information on various physical states along an evolutionary path.

4. Stellar pluralism. We have now at hand the main sources of pluralism in stellar classifications illustrated at the beginning of this paper by the display of the several kinds to which a given star may belong. Different investigation techniques focusing on different structural properties result in different, cross-cutting classifications. To illustrate this form of pluralism, consider UV based classifications and visible based classifications. The structural properties governing manifest properties in the UV include properties (mass loss and gradient velocity of stellar wind, among others) that do not govern manifest properties in the visible. So that stars, when observed in the UV, may divide up in a way different from the way they divide up in the HR diagram. Two stars classified in a same category of spectral type in the visible may have different spectra in the UV. ${ }^{13}$ This simply reflects the fact that two stars may have

13 Similar conclusions are reached when comparing spectral classifications in the visible and classifications based on stellar variability: it is not exceptional that two stars having the same spectral classification in the visible differ by their class of variability. 
similar structural properties governing visible manifest properties (say, similar temperature), but they may differ significantly by structural properties governing UV manifest properties (they may have different mass loss for instance).

The use of one domain of wavelength rather than another reflects specific epistemic interests. Astrophysicists interested in, say, the physics of stellar winds need a UV-based classification, whereas those studying evolved stars surrounded by dust rely on infrared-based classifications. And the same goes for the choice of the level of resolution of the taxonomic units: astrophysicists interested in stellar magnetic fields need high-resolution spectral classifications whereas those studying the chemical composition of stars in order to understand the overall chemical evolution of a galaxy are happy with standard resolution levels.

Stellar taxonomy should please pluralists on several grounds. Pluralists usually draw on the diversity of biological taxa to dismiss the monist quest for a unique correct way of classifying things (Dupré 1993, Kitcher 2001), whereas physics and chemistry are widely considered as monist-friendly (see for instance Ellis (2002) on the monist side and Slater (2005) on the pluralist side). Stellar taxonomy challenges this traditional partition by extending the domain of relevance of the pluralist claim beyond its usual domain, biology; what is more by adding to the pluralist's moneybag a significant bit of the traditional ally of the monist, namely the physical sciences.

Moreover, in light of similarities with the familiar case of the classification of chemical elements, stellar taxonomy invites us to reconsider the support this case traditionally brings to a monist standpoint on scientific classification. The main similarity between the two taxonomies is that in both cases, kind-membership is conferred by structural properties. But an important difference is that in the chemical case, a single microstructural property, to wit, nuclear charge, happens to be the overwhelming determinant of a large variety of chemical behaviors ${ }^{14}$, whereas we've just seen that no such single behavioral determinant is to be found in the stellar case.

This invites us to clearly distinguish between two claims: the claim that the appropriate kind-membership conditions are structural conditions, and the claim that there exists a single kind-membership condition (or set of) that is central to explaining a large variety of behaviors. Structuralism and monism happen to both hold for chemical elements (and, incidentally, neither hold for biological species), but the

14 As noted by Hendry $(2006,868)$, atomic weight, for instance, is a negligible factor, except for hydrogen where the isotope effect might be noticeable. 
stellar case shows us that they do not always go hand in hand ${ }^{15}$ : structuralism does not favor taxonomic monism over taxonomic pluralism. If chemistry is, on the face of it, hospitable to taxonomic monism, it is not because its kind-membership conditions are structural - if so, stellar taxonomy would also be monist-friendly. Inversely, a case for taxonomic pluralism does not require showing that structural kind-membership conditions cannot be found (note that this is a strategy commonly used by proponents of species pluralism - see for instance Dupré 1981).

How then should one interpret the monist-friendliness of the chemical element case? Stellar taxonomy draws our attention on a central part of the answer, by putting to the fore what is admittedly much less manifest in other domains of the physical sciences, to wit, the interest-dependency of a taxonomic system. We've just seen that different, cross-cutting taxonomic systems respond to different epistemic interests about stars. Is this interest-dependency specific to astrophysics and an exception in the physical sciences? On the face of it, the classification of chemical elements seems rather immune to interest-dependency: nuclear charge does not have any serious competitor as a grouping criterion for chemical elements, which would respond to alternative epistemic interests. For all that, this consensus should not be interpreted as vindicating that the periodic table constitutes an interest-free classification of the chemical elements. It can be argued that it rather reflects the fact that, as Hendry aptly emphasizes when contrasting chemistry with biology: "the interests that govern its classifications are more unified [...]" $(2006,865)$. For Hendry $(2006,874)$, the presence of "modal intentions" is what unifies the epistemic interests of the chemists. Dupré $(2006,30)$ also emphasizes the specific unified aims of chemistry as grounds for its taxonomic monist-friendliness. By contrast, no such unifying character is to be found in the epistemic interests scientists have in living organisms. Hence the much discussed interest-dependency and resulting pluralism of the groupings of living organisms. Population ecologists, for instance, do not group them according to the same parameters than biologists do. And even within biology, various theoretical perspectives notoriously translate into various grouping criteria defining distinct concepts of species, with the "Biological Species Concept" and the "Phylogenetic

\footnotetext{
15 This association between structuralism and monism is often made, not only by monists such as Wilkerson 1993, but also by pluralists (see for instance Slater 2005).
} 
Concept" being the most discussed ones ${ }^{16}$. But to make a case for the interestdependency of classifications in the physical sciences, pluralists have had so far to contend themselves with imaginary "fantasies" in which classifiers would have come up with different taxonomic systems, had they started with alternative or less unified epistemic interests. ${ }^{17}$ The good news for such pluralists is that astrophysics provides them with an actual case in the physical sciences to support their contentions that classifications are also interest-dependent in this domain.

Another ground of comfort for pluralists is that stellar pluralism appears as a much less controversial ally than their traditional one. By contrast with species pluralism, there is no controversy about the permanent character of stellar pluralism. Whereas people disagree on whether biological taxonomy should aim at identifying a single correct species concept ${ }^{18}$, astrophysicists do not aim at coming up with a unique set of kind-membership conditions that would ground a unique correct way of classifying stars. On the contrary, they explicitly admit that a complete knowledge of the physical nature and the evolution of stars requires different, cross-cutting taxonomic systems, each depending on the technique of observation used and the type of physical processes the technique gives access to. ${ }^{19}$ And indeed we have seen, when skimming through the history of stellar taxonomy, that the more mature stellar astrophysics has become, the more pluralist its taxonomy. So that monists could

16 For a recent overview of the lively discussions about species pluralism, see Ereshefsky 2007.

$17 \quad$ Here's how the fantasy may go: "Beings with different constitutions or with similar constitutions but different interests might begin with an alternative collection of manifest features, and their inquiries might single out alternative underlying microphysical structures, causally implicated in the cluster of characteristics that concerned them. Or perhaps the common causal factors would not be microphysical at all. Even in the case of the physical sciences, the alleged objective similarities carry a tacit relativization to our capacities and our interests" (Kitcher 2001, 50-51).

18 See again Ereshefsky 2007 for a review of the arguments exchanged by monists and pluralists on the desirability of a single species concept.

19 Here's how a specialist in stellar classification expresses the fruitfulness of crosscutting classifications: "The success, however, of classifications in region beyond the traditional MK region (i.e. UV or near IR) comes when these extensions, at least in the first instance, are kept completely autonomous from the MK system, i.e. the specimen are allowed to find their own order. This allows the (possibly different) classification from each wavelength region to reflect the different information about the layers in a stellar atmosphere, which may be only weakly coupled. When the classifications are compared, then the insight can begin." (Corbally, 1994)

At this point the monist could admittedly still claim that astrophysicists should not embrace pluralism, for they have yet to discover some more fundamental structural properties that would play a unifying role, but that would be at the cost of rejecting a huge chunk of solidly-established physics (a rather unappealing move). 
hardly deny that astrophysics support taxonomic pluralism on the grounds that it is just too an immature and messy science to be a reliable source for philosophical arguments.

The main upshot of my analysis of the grouping of stars is twofold. First, by challenging the idea (still widespread even among pluralists) that one domain at least, the physical sciences, is hospitable to interest-free, monistic classifications, stellar taxonomy further undermines the monist thesis (already significantly weakened by arguments drawing on the diversity of biological taxa) that there exists only one correct way of classifying things that science aims at discovering. Second, it shows that one may have several cross-cutting ways of grouping things all based on the same type of kind-membership condition, to wit, structural. In other words, stellar pluralism is less promiscuous, as regards kind-membership conditions, than taxonomic pluralism about living organisms, and therefore less prone to monist objections. For instance, Wilkerson's attack against Dupré's promiscuous realism on the grounds that too many of the kinds accepted by Dupré do not lend themselves to "serious scientific investigations" (Wilkerson 1993, 14), is irrelevant to the stellar case. Being all based on structural properties, stellar classifications do sort out stars into kinds that lend themselves to causal explanations and predictions. By contrast with the classifications of living organisms, they do not include any "useful system of classification" (Wilkerson 1993, 14) where any kind-membership condition goes, as long as it responds to peculiar practical or epistemic needs (as diverse as those of cookers, taxidermists, gardeners or professional biologists). For all that, are stellar kinds natural kinds?

5. Are stellar kinds natural kinds? The issue takes us back to the central question I left unanswered earlier, about the correct reading of the notion of "privileged" similarity parameters. It is time now to spell out the proper reading of the notion in the astrophysical context.

As suggested by Slater (2005), taxonomic monism may be split into two claims: a claim about classification - there exists one unique way of classifying things - and a metaphysical claim about the objectivity and the uniqueness of the distinctions demarcating natural kinds. Here's how Slater states that the latter does not imply the former: 
"The monist may thus grant that classification schemes can be as gerrymandered and parochial as you like (as need or interest dictates), so long as differences are sometimes ignored and sometimes regarded, rather than sometimes invented and sometimes discovered. Differences, the monist suggests, are discovered. Referential decisions may of course be made about which differences should be ignored in constructing a useful taxonomy of the world. Monism is a thesis about the actual kind distinctions (what natural kinds there are), not about the various ways we ignore those distinctions in cobbling together a workable system of classification. Classificatory pluralism does not entail metaphysical pluralism." (Slater 2005, 30)

So far only classificatory monism has been dismissed. Investigating which reading of the notion of "privileged" boundaries is valid in the stellar case will tell us whether, at least, metaphysical monism is tenable, or not. Metaphysical monism states that there exists some natural order, that is, some objective, mind-independent divisions that cut nature at its real joints in a unique way. In other words, the world comes prepacked with a unique set of objective divisions demarcating natural kinds. Note that metaphysical monism is stronger than realism. It does not only state that there exists real, mind-independent similarities and differences in nature; it also claims that there exists a uniquely privileged set of such similarities and differences, where "privileged" is conceived in an essentialist way. Sharing with Kripke (1972), Putnam (1975), Ellis (2002) and others a commitment to essentialism, here's how Wilkerson $(1993,5)$ sums up the thesis: "[...] there are many similarities and differences between things, one set is privileged because they are the real essences", the traditional candidates for essential properties being structural properties (for instance, genetic structures for biological species, molecular structures for chemical substances).

So does the stellar world come prepacked with a privileged set of objective divisions demarcating kinds defined by essential properties? Let us consider first whether essentialism is tenable about stellar kinds, before addressing the issue of realism. Essentialism traditionally requires the following (see for instance De Sousa 1984; Ellis 1996; Wilkerson 1993): kind-membership is conferred by possession of an essential property or properties, i.e. by a property or properties necessary and sufficient for membership of the kind in question. Moreover, essential properties are what determine law-like behaviors. Traditional essentialism also requires that a thing cannot belong to more than one natural kind (unless the kinds in question are hierarchically-nested kinds) and that natural kinds have sharp boundaries. In the 
stellar case, structural properties (temperature, density, etc.) are also the obvious candidates to the status of essential properties, for they are the type of properties that determine stellar behaviors. But the kinds they demarcate cannot count as natural kinds for at least two straightforward reasons: stellar kinds do not have sharp boundaries and a star may belong to more than one kind (not to mention taxonomic nomadism). Sticking to an essentialist conception of natural kinds would lead to the contention that there are far more stellar natural kinds than the stellar kinds currently demarcated by astrophysicists. Actually, in light of what has been said about the continuous character of taxonomic parameters and the lack of infimic kinds, the quest for essential properties inevitably leads us to "individualism" about natural kinds, that is, to count as many kinds of stars as there are stars! But individualism is a rather unappealing option ${ }^{20}$, to say the least, for a central motivation for the search of natural kinds is that they are supposed to be the subject of scientific laws. When you want to explain or predict the behavior of a thing, you identify the kind to which it belongs and apply the laws known to be governing the members of that kind. Saving an essentialist conception of stellar kind would thus come at the price of giving up on the economy of work that scientific generalizations governing the behavior of natural kinds are supposed to provide. The metaphysical monist may agree to pay this price, but such a radically monistic ontology not only seems a bit desperate but also utterly irrelevant to actual scientific practice.

On an essentialist, monistic reading of the term, our quest for "privileged" similarities and differences demarcating stellar kinds has thus proved fruitless. For want of such a unique set of "privileged" divisions, let us see now if, at least, a realist standpoint on divisions demarcating stellar kinds is tenable. Are differences demarcating stellar kinds objective? Are they discovered rather than conventionally marked by the classifier? We have to be careful here about what a negative answer means. Denying as I do that there are objective distinctions between stellar kinds does not mean that taxonomic features are not real, mind-independent features of the world. I do take temperature and density as objective features of the stellar world: no doubt, differences in terms of structural properties between individual stars are

$20 \quad$ Note that a monist such as Wilkerson $(1993,16)$ bites the bullet and admits that individualism is indeed a possibility for biological species defined in an essentialist way by their genetic structure, but immediately adds that it is very unlikely that we finished up with as many natural kinds as individuals. No such potential escape from individualism is available in astrophysics. 
discovered, rather than conventionally marked. But given the continuous variation of taxonomic parameters from one star to another, and the resulting vagueness and lack of infimic kinds, differences demarcating stellar kinds are not discovered but conventionally marked. Realism about stellar kinds is untenable.

So not only the stellar world does not come prepacked with a unique set of objective, privileged (in an essentialist sense) divisions, but also it does not come prepacked with objective divisions, tout court. In that respect, metaphysical monists and realists end up on the same boat: their only option to accommodate stellar kinds is to retreat to the unsavory individualist standpoint on natural kinds.

So, are stellar kinds natural kinds? Well, in light of the previous discussion, the answer is a ringing "no" on any realist reading of the notion, be it monist (essentialist) or pluralist (promiscuous realism à la Dupré) ${ }^{21}$. In other words, if they are "privileged" similarities and differences in the stellar world (and we have seen that, indeed, not any similarity parameters is taxonomically significant), it is not because they are objective, real ones, but because they define kinds that fullfill a useful role in scientific investigations. The appropriate reading of the term "privileged" in the astrophysical context is therefore an epistemological, interestdepending one. It is important to emphasize at this point that none of the previous philosophical lessons would need to be revised if the contingent limitations of choice of taxonomic parameters (discussed in section 3) could be overcome: all the candidates deemed epistemically significant, including those not used for practical reasons, vary continuously from one star to another, thereby leading to similar conclusions as regards resolution-dependency, vagueness, lack of infimic kinds, etc.

Given the correct reading of "privileged" in the astrophysical context, the next step would be to specify in what epistemological sense exactly stellar taxonomic parameters can be said to be privileged, in order to examine whether they favor one of the current non-essentialist doctrines that ground the fruitful role natural kinds are taken to play in scientific inquiries in terms of explanatory power (Laporte 2004) or categories allowing reliable predictions (Boyd 1999, Griffith 2004). My hunch is that

$21 \quad$ Here's how Dupré sums up his thesis: "The point is not that there are no real divisions in nature between kinds of things, divisions that are appropriate for a particular kind of inquiry, but what those divisions are will depend on what the enquiry is." $(2002,41)(M y$ italics). Only the last part of the thesis holds for stellar kinds: the appropriate divisions of the stellar world do indeed depend on the enquiry, but the divisions are conventionally marked, not real. 
stellar kinds will not be easily recruited by any of these doctrines, but establishing this point would require another paper devoted to a thorough analysis of the epistemic virtues of stellar kinds in terms of explanation, inductive prediction, etc. Meanwhile, one can at least raise the following question: if none of the current standpoints turned out to be hospitable to stellar kinds, would that be an embarrassment for those standpoints? This brings me to my brief concluding interrogative remarks on the relationship between actual scientific practice and philosophical doctrines of natural kinds.

When navigating through the intricate variety of currently competing doctrines of natural kinds, from strong, essentialist ones (Ellis 1996, 2002; Wilkerson 1993) to more or less weaker, non-essentialist ones (Boyd 1999; Laporte 2004; Dupré 1993, 2002; Griffith 2004), it is not always clear what their motivations and expectations are as regards existing scientific kinds. Is the relaxation of kind-membership conditions motivated by a desire to avoid ending up, in light of new scientific knowledge, with few candidates, or even no candidate at all, to the status of natural kind? Consider essentialism about biological species - for a long time the canonical examples of natural kinds. When traditional essentialism turned out to be untenable in light of post-Darwinian biological knowledge, was the development of more hospitable kindmembership conditions driven by a desire not to relieve biological species from their traditional status of natural kinds? If so, isn't tailoring a doctrine of natural kinds so that it includes one's favorite candidates (biological species being the most coveted ones) a bit circular? For what makes certain kinds paradigmatic examples of natural kinds to start with anyway? To come back to the stellar case, if none of the current standpoints on natural kinds can accommodate stellar kinds, what will be the appropriate attitude? Tailoring a weaker doctrine that accommodates this newcomer, or claiming: "so much for stellar kinds, they are just not natural kinds"? The point I want to make is simply the following: whatever the answers to those questions are, they will need to be justified. As newcomers in the field, what stellar kinds make thus vivid is the need, when discussing doctrines of natural kinds, for being explicit on general meta-commitments, to wit, commitments about what should be the constraints on those doctrines brought by the kinds defined and successfully used by practicing scientists. All the more reason for ending the philosophical disgrace of stellar kinds 
since Darwin's and Cournot's outdated verdicts and putting stars back on the agenda of discussions about natural kinds and classifications. 


\section{REFERENCES}

Boyd, Richard (1999), "Homeostasis, Species and Higher Taxa", in Robert A. Wilson (ed.), Species: New Interdisciplinary Essays. Cambridge, MA: MIT Press, 141-185.

Corbally, Christopher J. (1994), “The MK Process in Action Today" in Christopher J. Corbally, Richard O. Gray and Robert F. Garisson (eds.), The MK Process at 50 years, ASP Conference Series 60. San Fransisco: Astronomical Society of the Pacific, 237-246.

Cournot, Antoine-Augustin ([1851] 1975), Essai sur les fondements de nos connaissances et sur les caractères de la critique philosophique, Paris: Vrin.

Darwin, Charles ([1859] 1962), On the Origin of Species, New York: Collier Books.

Daston, Lorraine (2000), “The Coming into Being of Scientific Objects”, in L. Daston (ed), Biographies of Scientific Objects, Chicago: University of Chicago Press, 1-14.

De Sousa, Ronald (1984), "The Natural Shiftniness of Natural Kinds”, Canadian Journal of Philosophy, XIV, 4: 561-580.

DeVorkin, David H. (1978), "Steps toward the Hertzsprung-Russell diagram", Physics Today, March issue, 32-39.

Dupré, John (1981), "Natural Kinds and Biological Taxa", The Philosophical Review, 1:66-90.

Dupré, John (1993), The Disorder of Things. Cambridge, Mass.: Harvard Univeristy Press.

Dupré, John (2002), “Is 'Natural Kind' a Natural Kind Term?”, The Monist, 85: 2949.

Dupré, John (2006), “Scientific Classification”, Theory, Culture \& Society, 23:30-31.

Ellis, Brian (1996), "Natural Kinds and Natural Kind Reasoning”, in P. J. Riggs (ed), Natural Kinds, Laws of Nature and Scientific Methodology, Kluwer, 11-28.

Ellis, Brian (2002), The Philosophy of Nature: A Guide to the New Essentialism, Chesham: Acumen.

Ereshefsky, Marc (2007), "Species", The Stanford Encyclopedia of Philosophy (Summer 2007 Edition), Edward N. Zalta (ed.), URL = $<$ http://plato.stanford.edu/archives/sum2007/entries/species/>.

Ghiselin, Michael (1974), "A Radical Solution to the Species Problem", Systematic Zoology, 23:536-544. 
Gray, Richard O. (1994), "Precision classification" in Christopher J. Corbally, Richard O. Gray and Robert F. Garisson (eds.), The MK Process at 50 years, ASP Conference Series 60. San Fransisco: Astronomical Society of the Pacific, 75-83.

Griffiths, Paul E. (2004), "Emotions as Natural and Normative Kinds", Philosophy of Science, 71: 901-911.

Hendry, Robin F. (2006), "Elements, Compounds, and Other Chemical Kinds", Philosophy of Science, 73: 864-875.

Hull, David (1978), “A Matter of Individuality”, Philosophy of Science, 45:335-360.

Jaschek, Carlos, and Mercedes Jaschek (1990), The Classification of Stars, Cambridge, UK: Cambridge University Press.

Kitcher, Philip (2001), Science, Truth, and Democracy. Oxford: Oxford University Press.

Kripke, Saul (1972), "Naming and Necessity", in D. Davidson and G. Harman (eds) Semantics of Natural Language, Dordrecht: D. Reidel.

LaPorte, Joseph (2004), Natural Kinds and Conceptual Change, Cambridge, UK: Cambridge University Press.

Pannekoek, Anton (1961), A History of Astronomy, New York: Dover Publications.

Putnam, Hilary (1975), "The Meaning of 'Meaning"”, in Hilary Putnam, Philosophical Papers, vol. 2, Mind, Language, and Reality. Cambridge: Cambridge University Press, 215-271.

Ruphy, Stéphanie (1997), “L'art de classer les étoiles”, Ciel et Espace, 323: 66-70.

Ruphy, Stéphanie (2006), ““"Empiricism all the way down”: a defense of the valueneutrality of science in response to Helen Longino's "contextual empiricism"”, Perspectives on Science, 189-214.

Shapere, Dudley (1987), "Scientific Theories and Their Domains" in Janet A. Courany (ed.), Scientific Knowledge, Belmont: Wadsworth Publishing Company, 7794.

Slater, Matthew H. (2005), "Monism on the One Hand, Pluralism on the Other", Philosophy of Science, 72:22-42.

Wilkerson, T. E. (1993), "Species, Essences and the Names of Natural Kinds", The Philosophical Quaterly, 43:1-19. 